\title{
The reverse of the coin: Methotrexate and liver fibrosis in a group of psoriatic arthritis patients
}

\author{
Andreea Lili Barbulescu', Beatrice Andreea Chisalau², Cristina Dorina Pirvanescu², \\ Sineta Cristina Firulescu², Cristina Ene², Stefan Cristian Dinescu², Simona Banicioiu Covei², \\ Roxana Mihaela Dumitrascu², Carmen Nicoleta Oancea ${ }^{3}$, Bogdan Silviu Ungureanu, \\ Nicolae Dan Florescu ${ }^{4}$, Paulina Ciurea ${ }^{2}$, Florentin Ananu Vreju²

\footnotetext{
${ }^{1}$ Department of Pharmacology, University of Medicine and Pharmacy, Craiova, Romania

${ }^{2}$ Department of Rheumatology, University of Medicine and Pharmacy, Craiova, Romania
} \\ ${ }^{3}$ Department of Analytical Chemistry, University of Medicine and Pharmacy, Craiova, Romania \\ ${ }^{4}$ Department of Gastroenterology, University of Medicine and Pharmacy, Craiova, Romania
}

\begin{abstract}
Objectives. We aimed to assess the presence of liver fibrosis using transient elastography in a group of PsA patients, compared to controls, and its possible relationship with MTX treatment.

Methods. We performed a prospective study on 41 patients diagnosed with PsA admitted to the Rheumatology Department of the Emergency County Hospital Craiova and a control group, of 20 sex and age-matched subjects. Patients data included demographic, clinical and laboratory parameters and were collected from every patient in accordance to the study protocol. Liver stiffness was evaluated using transient elastograhy, by an experienced investigator, using Fibroscan system (Echosens, Paris, France).

Results. After assessing liver fibrosis (LF) by transient elastography, we obtained statistically significant values compared to controls $(6.04+1.65 \mathrm{kPa}$ vs $4.16+0.93 \mathrm{kPa}, \mathrm{p}=0.002)$; liver fibrosis was found in 11 patients $(26.81 \%)$, 9 of them undergoing MTX treatment.

Analyzing the impact of MTX treatment, we found statistically significant values between the two groups $(6.04+1.65$ $\mathrm{kPa}$ for MTX treated group vs $5.35+1.30 \mathrm{kPa}, \mathrm{p}=0.03$ for non-MTX patients). Analyzing the differences between patients with/without liver fibrosis, we reckoned the following data: no differences for sex, age, alcohol consumption, hypertension, diabetes or hepatic function; significant differences were observed for BMI, dyslipidemia and fatty liver.

Conclusions. Hepatic involvement, especially liver fibrosis might be underestimated in PsA patients, and it is necessary to be properly and periodically evaluated in order to prevent further complications and maintain the efficient and safe dose of MTX, the first line therapeutic agent chosen for these patients. Non invasive hepatic imaging methods, such as transient elastography should certainly be considered for screening, along with a proper monitoring of comorbidities.
\end{abstract}

Keywords: psoriatic arthritis, liver fibrosis, methotrexate, transient elastography

\section{INTRODUCTION}

Methotrexate (MTX) is the main therapeutic choice in inflammatory rheumatic diseases, such as rheumatoid arthritis (RA) or psoriatic arthritis (PsA) $[1,2]$. Long term treatment with low doses, used in rheumatology, is usually associated with mild side effects but occasionally, potentially severe events may appear, including hepatic toxicity. Liver involvement is histologically revealed by the presence of steatosis, stellate cells hypertrophy, hepatocyte anisonucleosis and fibrosis. Liver fibrosis has been reported related to MTX treatment in different percentages, between 0 and $33 \%$, and there are several studies that evaluated its prevalence in PsA [2-8]. Patients with PsA who develop MTX induced-liver injury after long term therapy, often have related risk factors for fatty liver disease, such as high alcohol intake, obesity, diabetes and administration of other potentially hepa- 
totoxic agents. Periodic evaluation of the parameters related to hepatic function (aminotransferases, albumin, alkaline phosphatase etc.) doesn't offer sufficient data regarding liver fibrosis and their values may be found in normal ranges, despite the presence of this process [8-11]. The golden standard investigation is considered to be the liver biopsy, but it may be associated with significant morbidity and mortality. As methotrexate-induced liver injury is not frequent in our current practice, the risk/benefit ratio of liver biopsy has been questioned [12,13]. Several new technologies have been developed in order to establish the diagnosis of chronic liver disease, including transient elastography (TE), a type of shear wave ultrasound elastography, using Fibroscan system that measures the speed of shear waves to estimate hepatic tissue stiffness. This is not considered an operator dependent technique, and it allows a fast evaluation, without producing any discomfort for the patient. Using such techniques, along with a proper periodic evaluation of each patient, individualized depending on the symptoms and associated conditions, enables an early detection of any possible adverse events that might appear during the treatment and allows an appropriate therapeutic management [7,14-16].

We aimed to assess the presence of liver fibrosis using transient elastography in a group of PsA patients, compared to controls, and its possible relationship with MTX treatment.

\section{MATERIAL AND METHODS}

We performed a prospective study on 41 patients diagnosed with PsA according to CASPAR criteria [17], admitted to the Rheumatology Department of the Emergency County Hospital Craiova, between October 2019-June 2020, and a control group, of 20 sex and age-matched subjects, without acute or chronic inflammatory diseases. Patients data included demographic, clinical and laboratory parameters and were collected from every patient in accordance to the study protocol. Disease Activity in PSoriatic Arthritis (DAPSA) and Psoriasis Area Severity Index (PASI) scores were calculated in all PsA patients. We excluded the patients with any recent infection, history of hepatitis B or C infection, treatment with hepatotoxic drugs, besides current treatment for the rheumatic condition, alcohol abuse, autoimmune live diseases or recently diagnosed neoplasia.

Liver stiffness was evaluated using transient elastograhy, by an experienced investigator, using Fi- broscan system (Echosens, Paris, France), composed of an ultrasound probe and a vibration attachment. Elasticity is calculated in kilopascals $(\mathrm{kPa})$ and is measured at a depth between 25 and $65 \mathrm{~mm}$, over an area of $1 \times 4 \mathrm{~cm}$; the results can vary between $2.5 \mathrm{kPa}$ and $75 \mathrm{kPa}$. A value over $7 \mathrm{kPa}$ is considered as hepatic fibrosis [6]. The patient positioning implies the right arm being in abduction, in order to increase the intercostal space and allow a better access to the right liver lobe. The measurements are performed at the cross section point of the medium axillar axis and the line that crossess the xiphoid process; there are 10 measurements to be performed, with the final result represented by their mean, in $\mathrm{kPa}$, and can be interpreted as the equivalent of METAVIR score (F0-F4). The machine displays the interquartile range (IQR) and the success rate for the examinations (the number of measures depending on the applied impulses). This method offers the advantage of being non invasive, a fast technique and without pain or discomfort for the patients.

The study was performed in accordance with the ethics and deonthology principles of the Helsinki Human Right's Declaration and aproved by the Local Ethics Comitee. All patients provided their written informed consent, after receiving a standard form which mentioned that the results would be used for research purposes.

For statistical analysis we used GraphPad Prism 5.5 and the results are presented as mean $\pm \mathrm{SD}$; in order to compare groups, we used t-test and One-way ANOVA, and for evaluating correlations, the Pearson/Spearman's coefficient. A level of $p<0.05$ was considered statistically significant.

\section{RESULTS}

We enrolled 41 PsA patients, with a mean age of $53.44+0.91$ years and a mean disease duration of $6.63+4.26$ years, and 20 controls, with similar demographic characteristics.

All of the patients received DMARD therapy, most of them MTX monotherapy (26 subjects); for 16 of the patients the management option included a combination of a synthetic and a biologic DMARD . Disease activity reckoned a mean value of $11.80+4.91$ (min.2, max. 25.8); for PASI, we calculated a mean of $15.32+7.12$, with limits between 0 and 28. Inflammatory markers were found with a mean value of $9.66+16.6 \mathrm{mg} / \mathrm{dl}$ for $\mathrm{C}$ reactive protein (CRP) and $23.27+15.59 \mathrm{~mm} / \mathrm{h}$ for erythrocyte sedimentation rate (ESR). 
Regarding comorbidities, we found dyslipidemia to be present in a percentage of 68.29 (28 patients), with altered values of total cholesterol and its fractions in all 28 and hypertriglyceridemia for 25 of them. High blood pressure was found in 21 patients (53.65\%), all of them treated and periodically monitored, diabetes in $8(19.52 \%)$ of them and non-Alcoholic fatty liver disease (NAFLD) in almost half of the patients (20).

The main characteristics of the study group are presented in table 1 .

TABLE 1. General data of the study group

\begin{tabular}{|l|l|}
\hline Patients (N) & 41 \\
\hline Female (N; \%) & $27(65.86)$ \\
\hline Male (N; \%) & $14(34.14)$ \\
\hline Age (years) & $54.34+0.91$ \\
\hline Disease duration (years) & $6.63+4.26)$ \\
\hline Type of psoriasis (N; \%) & \\
- Nail & $24(58.53)$ \\
- Skin & $35(85.36)$ \\
- Nail and skin & $24(58.53)$ \\
- Sine psoriasis & $5(12.19)$ \\
\hline Type of psoriatic arthritis (N; \%) & \\
- Peripheral & $32(78.04)$ \\
- Axial and peripheral & $9(21.95)$ \\
\hline CRP (mg/dl) & $9.66+16.6$ \\
\hline ESR (mm/h) & $23.27+15.59$ \\
\hline DAPSA & $11.80+4.91$ \\
\hline PASI & $15.32+7.12$ \\
\hline BMI (kg/m2) & $27.44+6.35$ \\
\hline UA (mg/dl) & $4.77+1.48$ \\
\hline Current medication & \\
- DMARD non biologic (N; \%) & $41(100)$ \\
- DMARD biologic (N; \%) & $16(39.02)$ \\
- MTX (N; \%) & 26 \\
\hline - Non-MTX DMARD (N; \%) & 15 \\
\hline NAFLD (N; \%) & $20(48.78)$ \\
\hline Hypertension (N;\%) & $21(53.65)$ \\
\hline Diabetes (N;\%) & $8(19.52)$ \\
\hline Smoking (N;\%) & $20(48.78)$ \\
\hline Dyslipidemia (N;\%) & $28(68.29)$ \\
\hline Alcohol consumption (N; \%) & $7(17.07 \%)$ \\
\hline BMI-body mass $\%$ \\
\hline
\end{tabular}

$B M I$ - body mass index; UA - uric acid

After assessing liver fibrosis (LF) by transient elastography, we obtained statistically significant values compared to controls $(6.04+1.65 \mathrm{kPa}$ vs 4.16 $+0.93 \mathrm{kPa}, \mathrm{p}=0.002$ ) (figure 1); liver fibrosis was found in 11 patients $(26.81 \%), 9$ of them undergoing MTX treatment.

Analyzing the impact of MTX treatment, we found statistically significant values between the two groups $(6.04+1.65 \mathrm{kPa}$ for MTX treated group vs $5.35+1.30 \mathrm{kPa}, \mathrm{p}=0.03$ for non-MTX patients) (figure 2).

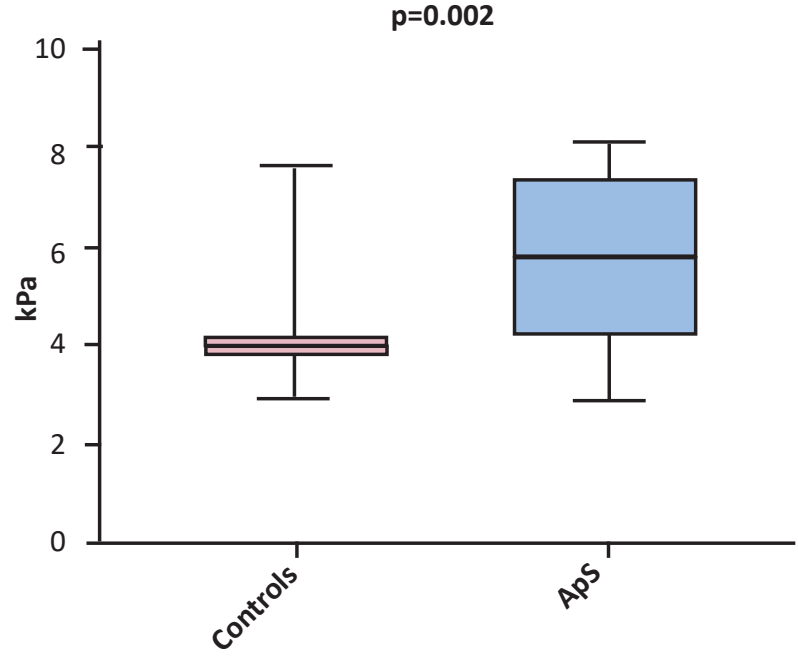

FIGURE 1. Liver stiffness for controls and patients

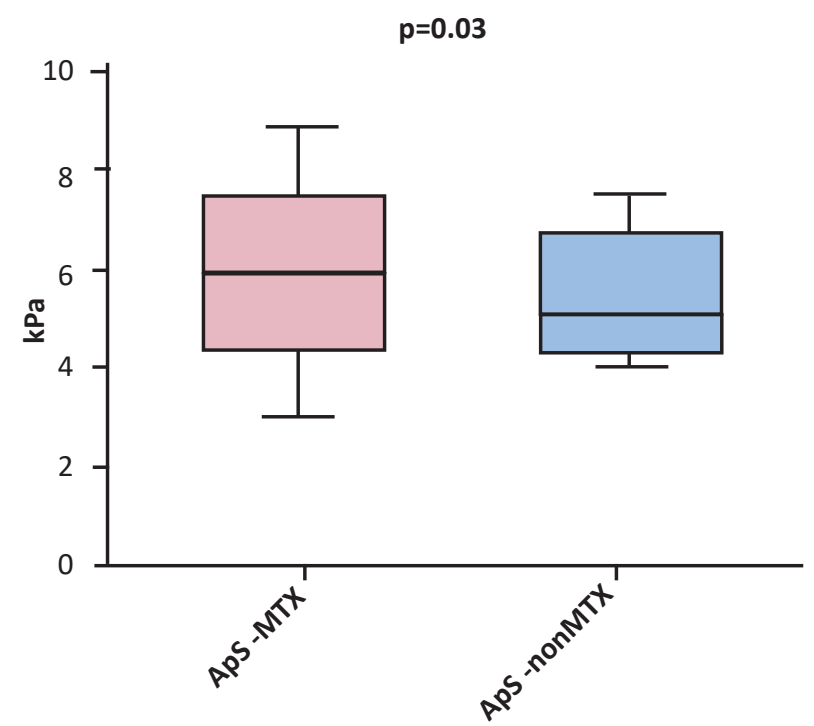

FIGURE 2. Liver stiffness depending on treatment (MTX/ non-MTX)

Analyzing the differences between patients with/ without liver fibrosis, we reckoned the following data: no differences for sex, age, alcohol consumption, hypertension, diabetes or hepatic function; significant differences were observed for BMI, dyslipidemia and fatty liver (table 2).

TABLE 2. Comparative parameters in the study group depending on the presence/absence of liver fibrosis

\begin{tabular}{|l|c|c|c|}
\hline & Fibrosis (11) & $\begin{array}{c}\text { Non fibrosis } \\
\text { (30) }\end{array}$ & $p$ \\
\hline Age (years) & $55.73+10.68$ & $50.55+11.12$ & 0.69 \\
\hline BMI (kg/m2) & $29.73+6.27$ & $26.60+6.27$ & 0.03 \\
\hline $\begin{array}{l}\text { Alcohol } \\
\text { consumption } \\
\text { (N; \%) }\end{array}$ & $2(18.18 \%)$ & $5(16.66 \%)$ & 0.08 \\
\hline NAFLD (N; \%) & $6(54.54 \%)$ & $14(46.46 \%)$ & 0.01 \\
\hline Diabetes (N; \%) & $2(18.18 \%)$ & $6(20 \%)$ & 0.68 \\
\hline $\begin{array}{l}\text { Hypertension } \\
\text { (N; \%) }\end{array}$ & $5(45.45 \%)$ & $16(53.33 \%)$ & 0.21 \\
\hline
\end{tabular}




\begin{tabular}{|l|c|c|c|}
\hline & Fibrosis (11) & $\begin{array}{c}\text { Non fibrosis } \\
\text { (30) }\end{array}$ & $p$ \\
\hline $\begin{array}{l}\text { Disease } \\
\text { duration (years) }\end{array}$ & $9.09+5.35$ & $4.36+2.99$ & 0.02 \\
\hline $\begin{array}{l}\text { MTX average } \\
\text { dose (mg/ } \\
\text { week) }\end{array}$ & $13.33+4.33$ & $13.67+5.16$ & 0.34 \\
\hline $\begin{array}{l}\text { MTX cumulative } \\
\text { dose (mg) }\end{array}$ & $3340+2769$ & $1834+1551$ & 0.15 \\
\hline DAPSA & $9.56+4.94$ & $12.62+4.62$ & 0.29 \\
\hline PASI & $13.11+8.34$ & $14.11+4.94$ & 0.59 \\
\hline ESR (mm/h) & $21.45+22.11$ & $20.80+10.35$ & 0.82 \\
\hline CRP (mg/dl) & $6.03+11.3$ & $5.86+5.28$ & 0.05 \\
\hline ALT (IU/l) & $23.7+13.8$ & $21.8+16.5$ & 0.58 \\
\hline AST (IU/l) & $21.9+17.3$ & $18.9+18.2$ & 0.56 \\
\hline ALP (IU/l) & $101.40+45.18$ & $96.23+25.12$ & 0.19 \\
\hline GGT (IU/l) & $58.20+20.34$ & $58.80+22$ & 0.28 \\
\hline $\begin{array}{l}\text { Blood glucose } \\
\text { (mg/dl) }\end{array}$ & $106.5+29.76$ & $101.3+31.46$ & 0.81 \\
\hline TC (mg/dl) & $252.7+64.30$ & $204.9+44.22$ & 0.007 \\
\hline LDL (mg/dl) & $115.9+21.10$ & $110.5+15.10$ & 0.02 \\
\hline HDL (mg/dl) & $69.57+22.92$ & $59.89+11.02$ & 0.09 \\
\hline TG (mg/dl) & $227.4+117.6$ & $129.4+61.18$ & 0.001 \\
\hline UA (mg/dl) & $7.30+3.96$ & $4.75+1.40$ & 0.32 \\
\hline BMI body mass index; $14+a 10 n i n e$ & \\
\hline
\end{tabular}

$B M I$ - body mass index; ALT - alanine aminotransferase;

AST - aspartate aminotransferase; GGT - gamma-glutamyl transferase;

$T C$ - total cholesterol; LDL - low-density lipoprotein;

HDL - high-density lipoprotein; TG - triglycerides; UA - uric acid

Analyzing the possible relationship between the presence of LF and different variables, we found a moderately positive correlation between LF and UA levels $(\mathrm{r}=0.50, \mathrm{p}<0.001)$, BMI $(\mathrm{r}=0.43, \mathrm{p}=0.03)$ and the presence of NAFLD $(r=0.51, p=0.02)$.

\section{DISCUSSION}

Generally, low dose MTX administered in inflammatory rheumatic conditions are not associated to severe side effects, but a part of them may be potentially severe, especially in the presence of comorbidities and related risk factors. Although several studies aimed to evaluate MTX hepatotoxicity, the direct drug impact is difficult to separate in the context of multiple therapeutic options and additional risk factors $[3,4,18,19]$.

Hepatic toxicity is still a special concern during MTX treatment and PsA patients are already exposed, due to an increased metabolic syndrome related liver disease risk, which limits therapeutic options and imposes careful and constant evaluation. Transient elastography has proved to be a useful and easily available method for this purpose $[4,6]$.

Enhanced liver stiffness was demonstrated in a percentage of 26.81 of the cases, significantly different compared to controls, most of them being under

MTX treatment. Our finding is similar to other reported studies that showed the presence of liver stiffness in different percentages between patients undergoing MTX treatment, significantly increased in case of PsA, compared to rheumatoid arthritis or Crohn disease $[3,6,20]$.

Early researches considered long term treatment with MTX a central risk factor for liver fibrosis and liver biopsy was mandatory in cases of psoriasis and PsA with cumulative doses over $1,500 \mathrm{mg}$ [21], observation that was not sustained by the results of later studies [16,22-26].

Still, a recently published review, that included 8 observational studies with over 400 patients with psoriasis, concluded that MTX treatment contributes to the development of liver fibrosis, without a clear association to the cumulative dose [8]. Other scientific reports have also reported a direct, positive correlation between the total cumulative dose and the presence and degree of liver fibrosis [8,22,24,27-29]. Our results firstly showed significant differences depending on MTX use, but carrying out further statistical analyses regarding the total dose and liver fibrosis, didn't point out noticeable differences of the cumulative dose between subjects with/without fibrosis. We are aware of some limitations of our study, related to small number of patients and the lack of the study group with PsA, but no MTX treatment, as the disease itself is associated to metabolic syndrome. Subsequently, different components of metabolic syndrome contribute to the development of hepatic disease, impacted by the concurrent use of MTX, and seemingly patients may develop fibrosis at lower cumulative doses in the presence of such comorbidities [29-31]. Indeed, the long term pro-inflammatory status in PsA patients contributes to overall risk of metabolic complications and can induce steatosis, condition that exposes the liver to inflammatory and oxidative injury, with consequent steatohepatitis and fibrosis [6,32]. Our data have also showed the presence of steatosis between PsA patients, in a higher percentage for the group of liver fibrosis, but not statistically different, possible to a relative reduced number of subjects.

Although our study included a relative low number of subjects, the data are significant and line with the ones published by several scientific publication and underline the necessity of a complete hepatic investigation, that can uncover a subclinical associated pathology and offer a better view of the possible outcome impact for these patients. 


\section{CONCLUSIONS}

Because PsA is a common inflammatory joint disease, associated with an increased risk of premature death, due to a frequent and high association with all the components of metabolic syndrome, it represents a challenge in the clinical practice, and it might be tricking regarding subclinical comorbidities and drug induced side effects.

Hepatic involvement, especially liver fibrosis might be underestimated, and it is necessary to be properly and periodically evaluated in order to prevent further complications and maintain the efficient and safe dose of MTX, the first line therapeutic agent chosen for these patients. Non-invasive hepatic imaging methods, such as transient elastography should certainly be considered for screening, along with a proper monitoring of comorbidities.

\section{Acknowledgements}

This paper is published under the frame of Research Project No.15/94-14.02.2020, University of Medicine and Pharmacy of Craiova.

Andreea Lili Barbulescu and Beatrice Andreea Chisalau share equal contributions to this paper.

Conflict of interest: none declared

\section{REFERENCES}

1. Rajitha P, Biswas R, Sabitha M, Jayakumar R. Methotrexate in the treatment of psoriasis and rheumatoid arthritis: Mechanistic insights, current issues and novel delivery approaches. Curr Pharm Des. 2017;23(24):3550-3566.

2. Dubey L, Chatterjee S, Ghosh A. Hepatic and hematological adverse effects of long-term low-dose methotrexate therapy in rheumatoid arthritis: An observational study. Indian J Pharmacol. 2016;48(5):591-594.

3. Laharie D, Terrebonne E, Vergniol J, Chanteloup E, Chabrun E, Couzigou P, et al. The liver and methotrexate. Gastroenterol Clin Biol. 2008;32:134-142.

4. Ledingham J, Gullick N, Irving K, Gorodkin R, Aris M, Burke J, Gordon P, Christidis D, Galloway S, Hayes E, Jeffries A, Mercer S, Mooney J, van Leuven S, Galloway J; BSR and BHPR Standards, Guidelines and Audit Working Group. BSR and BHPR guideline for the prescription and monitoring of non-biologic diseasemodifying anti-rheumatic drugs. Rheumatology (Oxford). 2017; 56(6):865-868

5. European Association for the Study of the Liver. EASLE-ALEH. Clinical practice guidelines: non-invasive tests for evaluation of liver disease severity and prognosis. J Hepatol. 2015; 63:237-264.

6. Laharie D, Seneschal J, Schaeverbeke T, et al. Assessment of liver fibrosis with transient elastography and FibroTest in patients treated with methotrexate for chronic inflammatory diseases: a case-control study. J Hepatol. 2010; 53:1035-1040.

7. Berends MA, Snoek J, de Jong EM, et al. Liver injury in long-erm methotrexate treatment in psoriasis is relatively infrequent. Aliment Pharmacol Ther. 2006; 24:805-811

8. Maybury CM, Jabbar-Lopez ZK, Wong T, Dhillon AP, Barker JN, Smith $\mathrm{CH}$. Methotrexate and liver fibrosis in people with psoriasis: a systematic review of observational studies. Br J Dermatol. 2014; 171:17-29.

9. Cuellar ML, LR Espinoza LR. Methotrexate use in psoriasis and psoriatic arthritis. Rheum Dis Clin North Am. 1997;23(4):797-809.

10. Weiyu Ye 1, Laura C Coates 2. Should Methotrexate Have Any Place in the Treatment of Psoriatic Arthritis? Rheum Dis Clin North Am. 2019;45(3):325-339.

11. M Cutolo 1, B Seriolo, C Pizzorni, C Craviotto, A Sulli. Methotrexate in psoriatic arthritis. Clin Exp Rheumatol. Nov-Dec 2002;20(6 Suppl 28): $\$ 76-80$

12. Cadranel J, Rufat $P$, Degos F. Practices of liver biopsy in France: results of a prospective nationwide survey. For the Group of Epidemiology of the French Association for the Study of the Liver (AFEF). Hepatology 2000;32:477-481.

13. Bravo AA, Sheth SG, Chopra S. Liver biopsy. N Engl J Med 2001;344:495-500.

14. European Association for the Study of the Liver. EASLE-ALEH. Clinical practice guidelines: non-invasive tests for evaluation of liver disease severity and prognosis. J Hepatol. 2015;63:237-264.
15. Cheng HS, Rademaker M. Monitoring methotrexate-induced liver fibrosis in patients with psoriasis: utility of transient elastography. Psoriasis (Auckl). 2018;8:21-29.

16. Berends MA, Snoek J, de Jong EM, et al. Biochemical and biophysical assessment of MTX-induced liver fibrosis in psoriasis patients: fibrotest predicts the presence and fibroscan predicts the absence of significant liver fibrosis. Liver Inter. 2007;27:639-645.

17. Taylor W, Gladman D, Helliwell P, Marchesoni A, Mease P, Mielants $H$. Classification criteria for psoriatic arthritis: development of new criteria from a large international study. Arthritis Rheum. 2006, 54(8):2665-2673.

18. Visser K, van der Heijde D. Risk and management of liver toxicity during methotrexate treatment in rheumatoid and psoriatic arthritis: a systematic review of the literature. Clin Exp Rheumatol. 2009;27:1017-25.

19. Conway R, Low C, Coughlan RJ, O‘Donnell MJ, Carey JJ. Risk of liver injury among methotrexate users: a meta-analysis of randomised controlled trials. Semin Arthritis Rheum. 2015;45(2):156-62

20. Fleming KM, Aithal GP, Solaymani-Dodaran M, Card TR, West J. Incidence and prevalence of cirrhosis in the United Kingdom, 19922001: a general population-based study. J Hepatol. 2008;49:732738.

21. Roenigk Jr HH, Auerbach $\mathrm{R}$, Maibach $\mathrm{H}$, Weinstein $\mathrm{G}$, Lebwohl M. Methotrexate in psoriasis: consensus conference. J Am Acad Dermatol. 1998;38:478-485.

22. Kalb RE, Strober B, Weinstein G, Lebwohl M. Methotrexate and psoriasis: ional Psoriasis Foundation Consensus Conference. J Am Acad Dermatol. 2009;60:824-837.

23. Laharie D, Zerbib F, Adhoute X, Boue-Lahorgue X, Foucher J, Castera $L$, et al. Diagnosis of liver fibrosis by transient elastography (FibroScan) and noninvasive methods in Crohn's disease patients treated with methotrexate. Aliment Pharmacol Ther. 2006;23:16211628.

24. Aithal GP, Haugk B, Das S, Card T, Burt AD, Record CO. Monitoring methotrexate-induced hepatic fibrosis in patients with psoriasis: are serial liver biopsies justified? Aliment Pharmacol Ther. 2004;19:391-399.

25. Pavy S, Constantin A, Pham T, Gossec L, Maillefert JF, Cantagrel A, et al. Methotrexate therapy for rheumatoid arthritis: clinical practice guidelines based on published evidence and expert opinion. Joint Bone Spine. 2006;73:388-395.

26. Lambert CM, Sandhu S, Lochhead A, Hurst NP, McRorie E, Dhillon $\mathrm{V}$. Dose escalation of parenteral methotrexate in active rheumatoid arthritis that has been unresponsive to conventional doses of methotrexate: a randomized, controlled trial. Arthritis Rheum. 2004;50:364-371.

27. Fisher MC, Cronstein BN. Metaanalysis of methylenetetrahydrofolate reductase (MTHFR) polymorphisms affecting methotrexate toxicity. J Rheumatol 2009;36:539-545. 
28. Pongpit J, Porntharukchareon S, Kaewduang P, et al. Liver stiffness measurement in psoriasis: do metabolic or disease factors play the important role? Biomed Res Int. 2016;2016:7963972.

29. Lindsay K, Fraser AD, Layton A, Goodfield M, Gruss H, Gough A. Liver fibrosis in patients with psoriasis and psoriatic arthritis on long-term, high cumulative dose methotrexate therapy. Rheumatology (Oxford). 2009;48:569-572.

30. Rodriguez-Zuniga MJM, Garcia-Perdomo HA. Systematic review and meta-analysis of the association between psoriasis and metabolic syndrome. J Am Acad Dermatol. 2017;77(4):657-666. e658.

31. Rosenberg P, Urwitz H, Johannesson A, et al. Psoriasis patients with diabetes type 2 are at high risk of developing liver fibrosis during methotrexate treatment. J Hepatol. 2007;46:1111-1118.

32. Rabinowich L, Shibolet O. Drug induced steatohepatitis: an uncommon culprit of a common disease. Biomed Res Int. 2015;2015:168905. 